Paidéia, 2005, 15(30), 57-68

\title{
ESTILOS COGNITIVOS E COMPREENSÃO LEITORA EM UNIVERSITÁRIOS ${ }^{1}$
}

\author{
Rosana Maria Mohallem Martins ${ }^{2}$ \\ Acácia Aparecida Angeli dos Santos \\ Universidade São Francisco \\ Isabel Cristina Dib Bariani \\ Faculdade de Educação da UNICAMP
}

\begin{abstract}
Resumo: Este estudo objetivou averiguar a existência de relações entre as características de estilos cognitivos de universitários ingressantes e sua habilidade de compreensão leitora. Participaram 120 alunos de uma universidade particular, de cursos noturnos das áreas de exatas, humanas e biológicas. Os instrumentos utilizados foram a Escala de Avaliação de Estilos Cognitivos e um texto preparado segundo a técnica de Cloze. Os resultados indicaram que a maior parte dos universitários estudados apresentaram pontuações mais elevadas nos estilos divergente e reflexivo; as características holista e serialista aparecem em proporções similares. Houve diferença significativa entre estilo cognitivo e gênero, indicando que os homens são mais holistas do que as mulheres. Quanto à compreensão de leitura, não foram identificadas diferenças estatisticamente significativas, considerando os cursos e o gênero dos estudantes. Também, identificou-se uma correlação negativa, marginalmente significativa, entre a habilidade de compreensão de leitura e o estilo cognitivo serialista.
\end{abstract}

Palavras-chave: avaliação psicoeducacional; ensino superior; estilos cognitivos; leitura.

\section{THE COGNITIVE STYLES AND READING COMPREHENSION AMONG UNDERGRADUATES}

\begin{abstract}
This study aimed at verifying the existence of relationships between the characteristics of the freshmen students' cognitive styles and their reading comprehension abilities. The informants were 120 students, from a private university attending night courses in the exact, human and biological areas. The instruments used were the evaluative Scale of Cognitive Style and a text prepared according to Cloze's technique. The results showed that the majority of the students tend to be divergent and reflexive; there are holist and serial characteristics in a similar proportion. There was a significant difference between the cognitive style and gender showing that men are more holistic than women. As for the reading comprehension, it wasn't identified any significant statistical difference, considering the student's courses and gender. A marginally significant negative correlation, between the reading comprehension and the serial cognitive style was also identified.
\end{abstract}

Key-words: psychoeducational evaluation; higher education; cognitive styles; reading.

\section{Introdução}

Os estudos sobre os estilos cognitivos foram desenvolvidos com base em interesses nas diferenças individuais na capacidade de pensar, perceber,

\footnotetext{
${ }^{1}$ Artigo recebido para publicação em 23/08/2004; aceito em 28/01/ 2005.

Endereço para correspondência: Acácia A. Angeli dos Santos, Universidade São Francisco - Campus Itatiba, Programa de Pós-graduação em Psicologia, R. Alexandre Rodrigues Barbosa, 45, 13251040, Itatiba-SP, E-mail: acacia.santos@saofrancisco.edu.br
}

lembrar de fatos e situações e resolver problemas. Santos, Bariani e Cerqueira (2000) afirmam que, desde o século IX, estudiosos já tinham interesse pelas variações individuais nos modos de pensamento, porém a expressão "estilo cognitivo" foi usada pela primeira vez por Allport em 1937. Esse autor considerava que todas as pessoas tinham tendências ou predisposições cognitivas e afetivas, que seriam os modos básicos para atuar e pensar e que determinariam as percepções e os julgamentos, sendo denominados de estilos cognitivos (Allport, 1973). 


\section{Rosana Maria Mohallem Martins}

O tema foi alvo de pesquisas durante a década de 60 e início da década de 70, no século XX. Vários estudiosos dedicaram-se ao assunto, porém, devido às inconsistências em relação à definição do construto, houve um declínio nas pesquisas sobre ele. A partir dos anos 80, os estudos sobre o tema foram retomados e passaram a focalizar os aspectos cognitivos, os de personalidade, bem como a provável origem genética das diferenças individuais (Lemes, 1998; Santos, Bariani \& Cerqueira, 2000).

Messick (1984) destaca que, embora exista uma variedade de definições, o ponto em comum nessas concepções indica que os estilos cognitivos não implicam em níveis de habilidade, capacidade ou inteligência. Assim, tratam do modo preferencial de alguém usar habilidades, não havendo estilos bons ou maus, apenas, diferentes estilos. De forma abrangente, os estilos cognitivos são entendidos como diferenças individuais na organização cognitiva, sendo vistos como mediadores entre habilidade e personalidade. Afetam o funcionamento metacognitivo, organizando e controlando o processamento de informação e as respostas emocionais.

Muitos dos estudiosos do tema limitam suas pesquisas a alguns tipos de estilos, não tratando simultaneamente da totalidade dos que já foram descritos na literatura (Buela-Casal, Carretero-Dios \& Santos-Roig, 2001; Messick, 1984; Riding, 1997; Santos, Bariani \& Cerqueira, 2000, entre outros). Em razão disso, foram escolhidos para o presente estudo alguns dos estilos referidos, a saber, o impulsivo/reflexivo, o convergente/divergente e o holista/serialista.

O estilo impulsivo está relacionado à tendência para dar respostas sem premeditação suficiente. Indivíduos impulsivos oferecem soluções muito rápidas aos problemas, sem consideração adequada da provável precisão das mesmas. As pessoas com esse estilo costumam aceitar a primeira hipótese elaborada sem dar maior importância à sua exatidão ou adequação e apresentam um melhor desempenho em tarefas que requerem processos globais. No estilo reflexivo considera-se a tendência para examinar e refletir sobre as possibilidades de soluções alternativas, analisando e diferenciando uma configuração complexa de estímulos em suas partes componentes. Pessoas com esse estilo tendem a considerar os méritos de suas hipóteses frente a determinadas tarefas, faz várias análises, eliminando e desconsiderando aquelas possivelmente errôneas. Os indivíduos têm um melhor desempenho em tarefas que requerem processos detalhados, seus pensamentos são mais organizados, seqüenciados e há ponderação prévia para suas respostas (Buela-Casal, Carretero-Dios \& Santos-Roig, 2001; Sternberg \& Grigorenko, 1997).

O estilo convergente relaciona-se com o pensamento lógico e com o raciocínio. As pessoas com esse estilo são hábeis em lidar com problemas que requerem uma clara resposta convencional, ou seja, uma solução correta, com base nas informações fornecidas. Preferem problemas formais e tarefas estruturadas, que demandam mais habilidades lógicas. São inibidos emocionalmente, sendo identificados como mais conformistas, disciplinados e conservadores. Já o estilo divergente é associado à criatividade, a respostas imaginativas, originais e fluentes. Os indivíduos preferem problemas informais, sendo hábeis em tratar com aqueles que demandam a generalização de várias respostas igualmente aceitáveis, cuja ênfase está na quantidade, variedade e originalidade das respostas. Socialmente são considerados irritados e até ameaçadores (Bariani, Sisto \& Santos, 2000).

Os holistas dão maior ênfase ao contexto global desde o início de uma tarefa; preferem examinar uma grande quantidade de dados, buscando padrões e relações entre eles. Usam hipóteses mais complexas, às quais combinam diversos dados. Já os serialistas dão maior ênfase em tópicos separados e em seqüências lógicas, buscando, posteriormente, padrões e relações no processo, para confirmar ou não suas hipóteses. Elegem hipóteses mais simples e uma abordagem lógico-linear, ou seja, de uma hipótese para a próxima, trabalhando passo a passo (Jones, 1997).

Diante de várias definições que não encontravam consistência nos resultados de pesquisas, alguns autores, durante a década de 90, empregaram instrumentos variados, buscando evidências de validade para o construto estilo cognitivo. Entre esses estudos, destaca-se o de Riding (1997), que demonstrou que os estilos de uma mesma dimensão devem ser independentes um do outro. Assim, por exemplo, uma pessoa deve ser convergente ou divergente, não tendo as características dos dois estilos da mesma dimensão. 
De acordo com Witkin e Goodenough (1991), o indivíduo teria estilo "fixo", ou seja, apresentaria predominância das características de apenas um dos estilos cognitivos que compõem uma dimensão. O estudo de Bariani, Sisto e Santos (2000), cujo objetivo foi o de validar a Escala de Avaliação de Estilos Cognitivo também mostrou correlação inversa entre as dimensões, confirmando que os estilos cognitivos são bipolares.

Entretanto, alguns estudos mostram que os estilos cognitivos nem sempre aparecem como bipolares, ou seja, suas dimensões não são correlacionadas negativamente. No estudo de Bariani (1998), apenas algumas análises evidenciaram a tendência das dimensões a se correlacionarem negativamente. No entanto, a autora apresenta hipóteses explicativas para esse fato. Uma das hipóteses relaciona-se com a questão das imbricações, ou seja, os estilos não se apresentam de forma pura, mas se configuram pela mesclagem de diferentes estilos. A outra hipótese refere-se à versatilidade dos estilos cognitivos, que denotaria o desenvolvimento dos diferentes pólos das dimensões.

Messick (1984) defende a idéia de que os estilos cognitivos podem se correlacionar positivamente, sendo flexíveis e não rígidos. Afirma que o método de criar uma dimensão estatisticamente bipolar não é aplicável somente quando os dois pólos se correlacionam negativamente, mas também quando eles variam independentemente ou, até mesmo, quando apresentam correlações positivas baixas. Um estilo cognitivo representa um equilíbrio relativo dos meios alternativos pelos quais um indivíduo processa a informação e organiza a experiência; a presença de uma dimensão de estilo não significa a ausência da outra. Isso sugere que pessoas que apresentam ambas as tendências em graus variados podem ser consideradas como flexíveis. Essa flexibilidade seria detectada quando um indivíduo apresentasse médias altas nas duas dimensões opostas de um estilo (por exemplo, holista/serialista), diversamente daquele que tivesse pontuação baixa em ambas as dimensões.

A teoria nomeada de Auto-Direção Mental foi proposta por Sternberg e Grigorenko (1997), defendendo a idéia de que todas as pessoas, de certa maneira, possuem todos os estilos. Segundo essa teoria, o que difere nos indivíduos seria a força das prefe- rências e os tipos de tarefas e situações que evocam essas preferências. Os autores consideram que, em geral, os estudos rotulam as crianças como impulsivas ou reflexivas baseados em valores de escalas numéricas. Para eles, qualquer pessoa pode agir reflexivamente ou impulsivamente, dependendo da situação. No presente estudo pretende-se focalizar a situação educacional como contexto no qual os estilos se manifestam, produzindo efeitos no processo de aprendizagem e, mais especificamente, na compreensão da leitura.

\section{Estilos cognitivos no contexto de escolarização}

Em função dos estilos, os alunos podem ter mais ou menos facilidade diante de alguns métodos de ensino, alguns assuntos, enfim, podem revelar melhor desempenho em diferentes situações. Conhecendo o próprio estilo, o aluno pode perceber em que situação aprende melhor e o professor pode identificar em que situação consegue ensinar, atingindo um número maior de alunos. Alunos e professores, conhecendo o próprio estilo, podem desenvolver estratégias que favoreçam o desenvolvimento de outros estilos cognitivos, adequando-se a diversas realidades (Riding \& Wigley, 1997).

Considera-se que o desempenho em uma determinada tarefa é favorecido pelo tipo de estilo (Riding, 1997). Assim sendo, um maior conhecimento sobre os estilos cognitivos poderia favorecer processos de ensino e aprendizagem mais adequados, respeitando as características individuais dos alunos. Tal fato fica evidente nos estudos desenvolvidos por Bariani e Santos (2000) e por Pitta, Santos, Escher e Bariani (2000) com alunos de psicologia, mostrando como os estilos podem favorecer o interesse por atividades de pesquisa.

Nesse sentido, seria altamente desejável um maior investimento em pesquisas que focalizem variáveis psicológicas, como os estilos cognitivos, uma vez que podem interferir na aprendizagem. No entanto, esse construto tem sido pouco investigado no Brasil. Em uma pesquisa desenvolvida recentemente, Santos, Sisto e Martins (2003) dão destaque para algumas das possíveis relações existentes entre as dimensões dos estilos cognitivos e traços de personalidade, tendo como sujeitos universitários ingressantes. 


\section{Rosana Maria Mohallem Martins}

Voltando-se para questões específicas do contexto educacional, verifica-se que a compreensão de leitura é uma das mais enfatizadas, tendo em vista sua importância para o processo de aprendizagem. É crescente o número de pessoas que são alfabetizadas, mas que não compreendem o que lêem. Entre essas pessoas estão muitos estudantes que ingressam na universidade sem a necessária proficiência em compreensão da leitura. Pesquisas brasileiras e estrangeiras mostram o baixo nível de habilidade na compreensão de leitura em estudantes universitários (Abrahan \& Chapelle, 1992; Carelli \& Santos, 1998; Carpenter, Miyake \& Just, 1995; Oliveira, 1993; Pellegrini, 1996; Storey, 1997).

Em um curso superior aspira-se que o aluno seja capaz de ler segundo um propósito; criar suas próprias estratégias de compreensão, adequando-se às características do texto; sintetizar a informação a partir do texto e de sua própria experiência; assumir uma atitude avaliativa frente à informação; elaborar inferências referentes a causas e predição, e por fim, aplicar o conhecimento para resolução de problemas (Vicentelli, 1999). O sucesso em um curso universitário está relacionado com a maturidade de leitura do estudante, que inclui habilidades como compreensão, ritmo, concentração, flexibilidade, criticidade e criatividade. Santos $(1997)$ e Witter $(1997,1999)$ ressaltam a importância do fator compreensão, afirmando que a mesma não existe se não existir leitura, visto que é por meio dela que se pode dizer que o texto foi realmente lido.

A compreensão faz com que a leitura não seja apenas uma atividade mecânica de decodificação de palavras, sendo importante destacar que muitos fatores estão relacionados a ela. Alguns deles decorrem do contexto sócio-histórico-cultural em que a pessoa nasce e vive, incluindo sexo, idade, contexto familiar e escolaridade (Santos, 1994). Embora muitos aspectos relevantes para a compreensão da leitura sejam referentes a fatores externos ao indivíduo, o enfoque neste estudo será nos aspectos internos, em especial nos aspectos cognitivos relacionados à compreensão do texto.

A compreensão de leitura será aqui entendida com base nos pressupostos da psicologia cognitiva e, mais especificamente, na teoria do processamento humano de informações. Essa abordagem busca explicar os processos subjacentes do raciocínio humano, considerando as diferenças individuais e analisando a forma pela qual o indivíduo resolve problemas, memoriza elementos, entre outras atividades cognitivas. Nessa abordagem, a leitura é considerada como um processo complexo que envolve linguagem, memória, pensamento, inteligência e percepção. Mais ainda, é considerada como especialmente importante, por proporcionar o acesso ao léxico, que envolve a recuperação da palavra na memória permanente e a compreensão, que se refere à apreensão do conteúdo do texto como um todo. Perfetti (1992) e Sternberg (2000) afirmam que esses dois aspectos estão estreitamente relacionados à capacidade para a leitura.

Sendo uma habilidade complexa, a compreensão de leitura não é diretamente observável, por isso sua avaliação deve fundamentar-se em testes confiáveis. O Cloze vem sendo muito utilizado e tem se mostrado uma técnica bastante eficaz, tanto do ponto de vista prático, pela facilidade de elaboração, aplicação e correção, como do ponto de vista empírico, em razão dos altos índices de correlação positiva de seus resultados com o desempenho acadêmico (Bitar, 1989; Condemarín \& Milicic, 1988; Hannon \& Daneman, 2001; Helfeldt \& Henk, 1985; Santos, 1990).

A compreensão de leitura requerida pela técnica de Cloze envolve, entre outras variáveis, a habilidade do leitor em estabelecer relações entre os elementos do texto e a sua capacidade de desenvolver associações apropriadas entre o conhecimento prévio e a informação impressa (compreensão inferencial e lexical). Essas habilidades podem variar de indivíduo para indivíduo, considerando que o processamento da informação depende das características individuais. Valendo-se do Cloze como instrumento de avaliação, duas pesquisas recentes com universitários ingressantes, realizadas por Oliveira (2003) e Silva e Santos (2004) demonstraram que muitos alunos entram para um curso superior sem apresentar as habilidades de leitura necessárias para alcançar um bom desempenho acadêmico.

Mediante as considerações realizadas, podese hipotetizar que se cada pessoa apresenta formas intrínsecas de processar informações, de acordo com o seu estilo cognitivo, isso pode afetar o seu nível de compreensão de leitura. Com base nesse pressuposto, este trabalho propôs-se a investigar as ca- 
racterísticas de estilos cognitivos de universitários, bem como sua habilidade de compreensão de leitura. Além disso, buscou explorar a existência de eventuais relações entre os estilos cognitivos e a habilidade de compreensão de leitura, considerando variáveis referentes ao curso e gênero.

\section{Método}

\section{Participantes}

Fizeram parte do estudo 120 universitários ingressantes, de uma universidade particular do interior de São Paulo, de cursos noturnos de Administração ( $n=35)$, Análise de Sistemas ( $n=32)$ e Farmácia $(n=53)$. Optou-se por cursos que fizessem parte das diferentes áreas do conhecimento, respectivamente, da área de humanas, exatas e biológicas. A média de idade dos estudantes foi de 22,45 anos, sendo que a idade mínima foi de 17 anos e a máxima de 47 anos, com desvio padrão de 5,251. Em relação ao gênero, $50,8 \%$ dos estudantes eram do sexo masculino e 49,2 $\%$ do sexo feminino.

\section{Instrumentos}

\section{Escala de Avaliação de Estilos Cognitivos}

Escala do tipo Likert, construída e validada por Bariani, Sisto e Santos (2000), destinada à descrição dos estilos cognitivos preferenciais. O instrumento avalia três dimensões, impulsivo/reflexivo, convergente/divergente e holista/serialista. Para respondê-lo o sujeito deve indicar em que grau concorda, discorda ou se não tem opinião a respeito, colocando um "X" em umas das categorias: DT (discordo totalmente), D (discordo), I (indeciso), C (concordo), CT (concordo totalmente).

\section{Teste de Cloze}

O procedimento de Cloze foi proposto por Taylor (1953) e consiste em eliminar palavras de um texto escrito de, aproximadamente duzentos vocábulos, substituindo-as por um espaço vazio sublinhado. Esse espaço pode ser de tamanho sempre igual, como recomendado pelo autor, ou por um traço de tamanho proporcional ao da palavra retirada, como proposto por outros autores
(Bormuth, 1968; Henk, 1981; Rush \& Klare,1978).

Para descrever a habilidade de compreensão de leitura dos estudantes, foi utilizado um texto de Veríssimo (1995), com o título "Desentendimento". O texto de 200 palavras foi estruturado com omissão aleatória de todos os quintos vocábulos, num esquema de razão fixa, o que implicou em um total de 40 lacunas a serem preenchidas. A aplicação envolveu uma instrução de simples compreensão, que consistia em ler o texto todo, depois voltar ao início e preencher as lacunas com as palavras que o sujeito julgasse mais adequadas, levando em consideração o assunto apresentado.

\section{Critérios de Avaliação}

Para a avaliação da Escala de Estilos Cognitivos, foi calculada a média em cada estilo, somando os itens, de acordo com a categoria escolhida (DT / D / I / C / CT). Cada categoria de resposta tem uma pontuação que varia de 1 a 5 , sendo 1 para DT, 2 para o D, 3 para I, 4 para C e 5 para CT.

Para o Teste de Cloze, foi computado um ponto para cada resposta correta. A correção foi literal, existindo apenas uma única resposta válida e idêntica à palavra retirada do texto original. A pontuação final foi resultante da soma dos itens corretamente preenchidos até o máximo de 40 pontos, correspondente ao número de omissões. Vale destacar que o tipo de correção adotada é referendada por vários pesquisadores (Henk, 1981; Rush \& Klare, 1978; Santos, 1990, entre outros) que não encontraram diferenças significativas entre a pontuação obtida com a correção literal e a sinônima, quando se considera a média de acertos do grupo em geral e o objetivo é de diagnóstico do nível de compreensão, como no estudo aqui apresentado.

\section{Procedimento}

Inicialmente, foi solicitada, junto aos diretores dos cursos, permissão para a realização da pesquisa e ficaram combinados os dias e horários para a aplicação dos instrumentos. A aplicação foi coletiva, em duas sessões e sem tempo determinado, tendo participado apenas os alunos que assinaram o Termo de Consentimento Esclarecido. 


\section{Resultados}

Tendo em vista os objetivos pretendidos, os dados foram analisados com provas de estatística descritiva e inferencial, que permitissem a explora- ção das variáveis envolvidas. Assim, em relação aos estilos cognitivos, considerando a variável área de conhecimento, foram calculadas as médias e desvios-padrão de cada uma das dimensões da escala, tal como se observa na Tabela 1.

Tabela 1 - Média e desvio padrão de cada dimensão de estilo por curso.

\section{Dimensões $\quad$ Convergente Divergente Holista Serialista Impulsivo Reflexivo}

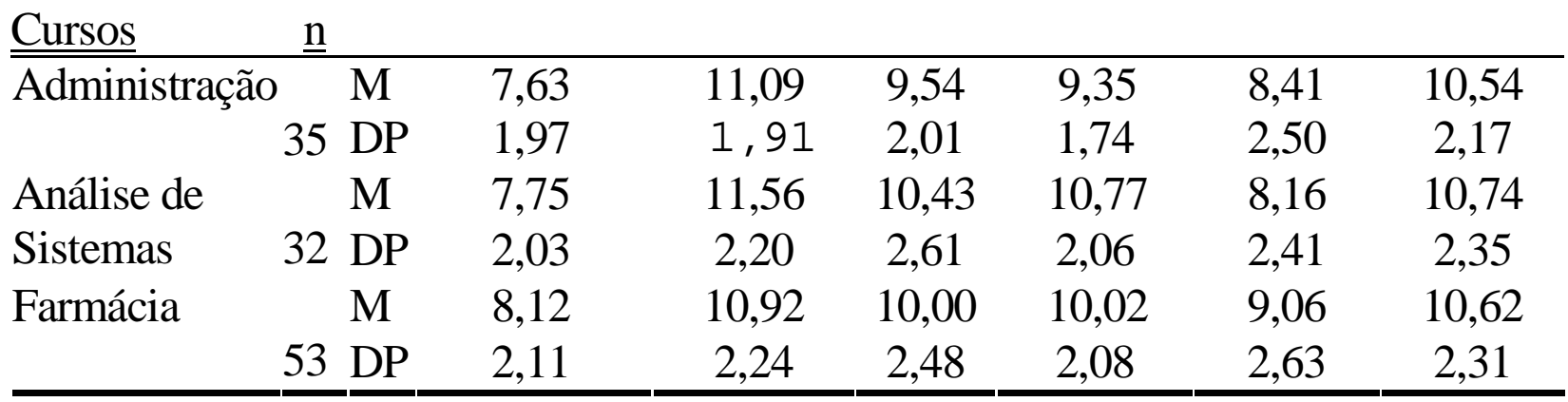

De modo geral, pode-se observar a maior média no estilo divergente $(11,56)$ nos estudantes do curso de Análise de Sistemas. A menor média obtida foi no estilo convergente $(7,63)$ entre os estudantes do curso de Administração. Para se comparar as médias por estilo entre os cursos, empregou-se a análise de variância, sendo constatada diferença apenas no estilo serialista. Com a aplicação da prova post-hoc de Tukey detectou-se que a pontuação média dos alunos do curso de Análise de Sistemas era significativamente superior à obtida pelos alunos do curso de Administração $[\underline{F}(2,118)=4,167$; $\mathrm{p}=0,01]$.

Para comparar os estilos cognitivos em relação ao gênero também foram calculados as médias e desvios-padrão. Ao lado disso, utilizou-se o teste $\underline{t}$ de Student para análise inferencial das diferenças entre as médias em cada um dos estilos, sendo os resultados obtidos apresentados na Tabela 2 . 
Estilos cognitivos e compreensão leitora 63

Tabela 2 - Média, desvio-padrão e comparação das dimensões dos estilos cognitivos por gênero (N=120).

\begin{tabular}{|c|c|c|c|c|c|}
\hline Dimensões & Gênero & $\underline{M}$ & $\underline{D P}$ & & $\underline{t}$ \\
\hline \multirow[t]{2}{*}{ Convergente } & $\begin{array}{c}M \text { a sculino } \\
(\underline{\mathrm{n}}=61)\end{array}$ & 7,89 & 2,050 & \multirow[t]{2}{*}{0,068} & \multirow[t]{2}{*}{0,946} \\
\hline & $\begin{array}{c}\text { F e m in in o } \\
(\underline{n}=59)\end{array}$ & 7,86 & 2,057 & & \\
\hline \multirow[t]{2}{*}{ Divergente } & $\begin{array}{c}M \text { asculino } \\
(\underline{n}=61)\end{array}$ & 11,28 & 2,303 & \multirow{2}{*}{\multicolumn{2}{|c|}{$0,7030,484$}} \\
\hline & $\begin{array}{c}\text { F e m in in o } \\
(\underline{n}=59)\end{array}$ & 11,00 & 1,954 & & \\
\hline \multirow[t]{2}{*}{ Holista } & $\begin{array}{c}\text { M a sculino } \\
(\underline{n}=61)\end{array}$ & 10,49 & 2,322 & \multirow[b]{2}{*}{2,426} & \multirow[b]{2}{*}{0,017} \\
\hline & $\begin{array}{c}\text { F e m in in o } \\
(\underline{n}=59)\end{array}$ & 9,44 & 2,353 & & \\
\hline \multirow[t]{2}{*}{ Serialista } & $\begin{array}{c}M \text { asculino } \\
(\underline{n}=61)\end{array}$ & 10,03 & 2,017 & \multirow[b]{2}{*}{0,043} & \multirow[b]{2}{*}{0,966} \\
\hline & $\begin{array}{c}\text { Fe m in in o } \\
(\underline{n}=59)\end{array}$ & 10,02 & 2,074 & & \\
\hline \multirow[t]{2}{*}{ Impulsivo } & $\begin{array}{c}\text { M a sculino } \\
(\underline{n}=61)\end{array}$ & 8,85 & 2,406 & \multirow[b]{2}{*}{0,967} & \multirow[b]{2}{*}{0,335} \\
\hline & $\begin{array}{c}\text { Fe m in in o } \\
(\underline{n}=59)\end{array}$ & 8,40 & 2,682 & & \\
\hline \multirow[t]{2}{*}{ Reflexivo } & $\begin{array}{l}\text { M a sculino } \\
(\underline{\mathrm{n}}=61)\end{array}$ & 10,42 & 2,287 & \multirow{2}{*}{$-1,037$} & \multirow[b]{2}{*}{0,302} \\
\hline & $\begin{array}{c}\text { Fe m in in o } \\
(\underline{n}=59)\end{array}$ & 10,85 & 2,242 & & \\
\hline
\end{tabular}

Os resultados apontaram que houve uma diferença estatisticamente significativa $(\mathrm{p}=0,017)$ no estilo holista. Tal diferença parece indicar que os homens têm esta dimensão mais acentuada do que as mulheres.

No que se refere à compreensão de leitura, as médias, os desvios-padrão e o número de sujeitos, por curso, estão apresentados na Tabela 3.

Tabela 3 - Estatísticas descritivas da habilidade de compreensão de leitura por curso e por gênero.

\begin{tabular}{lccccc}
\hline Cursos & $\underline{\mathrm{n}}$ & $\underline{\mathrm{M}}$ & $\underline{\mathrm{DP}}$ & $\begin{array}{c}\text { Pontos } \\
\text { mínimos }\end{array}$ & $\underline{\underline{\text { Pontos }}}$ \\
$\underline{\underline{\text { máximos }}}$ \\
Administração & 35 & 18,23 & 3,24 & 13 & 26 \\
Farmálise de Sistemas & 32 & 19,59 & 3,17 & 10 & 26 \\
Masculino & 53 & 18,89 & 3,48 & 12 & 26 \\
Feminino & 61 & 18,70 & 3,53 & 10 & 26 \\
\hline
\end{tabular}




\section{Rosana Maria Mohallem Martins}

Pelos resultados obtidos foram observadas pontuações médias muito semelhantes entre os diferentes cursos. A análise por meio do teste t de Student não identificou qualquer diferença estatisticamente significativa por curso ou por gênero.

Recorreu-se à prova de correlação para explorar possíveis relações entre as duas medidas obtidas, a saber, sobre os estilos cognitivos e a habilidade de compreensão de leitura no mesmo grupo de alunos. Na Tabela 4 estão os resultados dos índices obtidos.

Tabela 4 - Índice de correlação de Pearson $(r)$ e nível de significância $(p)$ entre os estilos cognitivos e a compreensão de leitura $(N=120)$.

\begin{tabular}{ccc}
\hline Dimensões & $\underline{\mathrm{r}}$ & $\underline{\mathrm{p}}$ \\
\hline Convergente & $-0,055$ & 0,551 \\
\hline Divergente & $-0,103$ & 0,268 \\
\hline Holista & $-0,043$ & 0,649 \\
\hline Serialista & $-0,172$ & 0,065 \\
\hline Impulsivo & 0,039 & 0,678 \\
\hline Reflexivo & $-0,124$ & 0,180 \\
\hline
\end{tabular}

Recorrendo-se à prova de correlação de Pearson, verificou-se um índice de correlação negativa $(\underline{\mathrm{r}}=-0,172)$ não muito alto, mas marginalmente significativo $(\mathrm{p}=$ 0,065 ), entre o estilo cognitivo serialista e o escore obtido no Cloze. Assim, detectou-se uma tendência de maior pontuação no estilo cognitivo serialista entre os estudantes com menor compreensão de leitura.

\section{Discussão}

Considerando os resultados, os alunos de todos os cursos, aqui estudados, tendem a ser divergentes e reflexivos, estilos nos quais obtiveram as notas mais altas. Estes dados são congruentes com os encontrados por Bariani (1998), que também detectou entre os universitários que estudou predominância do estilo divergente sobre o convergente e do reflexivo sobre o impulsivo.

Da mesma forma, nos estudos de Pitta, Santos, Escher e Bariani (2000) e de Bariani e Santos (2000), focalizando apenas estudantes de Psicologia, os resultados também mostraram pontuações médias mais elevadas nos estilos divergente e reflexivo. Como os achados do presente estudo, o terceiro estilo em que os alunos apresentaram pontuação mais alta foi o serialista.

Os resultados da análise de variância mostraram uma pontuação significativamente superior no estilo serialista para os alunos do curso de Análise de Sistemas, quando comparados aos alunos do curso de Administração. Já, no estudo de Bariani (1998), quando comparados estudantes de diferentes cursos, foram encontradas diferenças estatisticamente significativas nos estilos campo independente, impulsivo e reflexivo. Os alunos do curso de Arquitetura e Urbanismo, por exemplo, apresentaram escores maiores no estilo campo independente e escores menores no estilo reflexivo e os de Psicologia obtiveram médias menores no estilo impulsivo, em relação aos outros cursos. Apesar das diferenças encontradas serem significativas, a autora entendeu que os resultados deveriam ser olhados com cautela, sem atribuir as diferenças apenas ao caráter do curso freqüentado, visto que explicações alternativas, relacionadas ao gênero dos estudantes e às vivências na universidade poderiam ser responsáveis por tais diferenças. O mesmo cuidado deve ser tomado ao se ponderar sobre os resultados aqui encontrados, visto que as mesmas considerações levantadas por Bariani em 1998 se aplicam no presente estudo.

O resultado deste estudo tem pouco a corroborar com o que Riding (1997) aponta como uma das evidências de validade do construto de estilos cognitivos. O autor propõe que os estilos estão relacionados aos comportamentos observáveis, como por exemplo, as preferências por assunto. Portanto, seriam esperadas diferenças mais acentuadas entre os cursos, considerando que os indivíduos tenderiam a escolhê-los de acordo com suas formas de perceber e de processar informações. Na pesquisa que realizou verificou que os indivíduos que tinham o estilo cognitivo global (equivalente ao holista) foram mais bem avaliados nos cursos de Contabilidade e Informática, que requerem maior capacidade para uma visão do todo. Nos achados deste estudo, os alunos de Análise de Sistemas foram os que obtiveram também as pontuações mais altas no estilo holista, embora as diferenças encontradas não tenham sido estatisticamente significativas. 


\section{Estilos cognitivos e compreensão leitora 65}

Com relação às diferenças existentes nas pontuações dos estilos cognitivos entre os sexos, os resultados indicaram que os homens são mais holistas que as mulheres, ou seja, dão maior ênfase ao contexto global do que a tópicos separados de uma tarefa. A literatura estrangeira sobre essa questão diverge dos resultados aqui encontrados, visto que no estudo de Riding, Burton, Rees e Sharratt, citado por Riding (1997), não apareceram diferenças em relação ao gênero, com respeito às dimensões do estilo cognitivo. Já o estudo de Bariani (1998) indicou que as mulheres, quando comparadas aos homens, tendem a ser menos divergentes e mais reflexivas. A autora analisa essa tendência, refletindo sobre as influências sociais no papel sexual, que indicam como os indivíduos de cada gênero devem se portar. Essa tendência parece se originar muito mais de características culturais, não podendo ser atribuída a fatores biológicos. Nesse sentido, pode-se supor que tais diferenças podem ter impacto nos estilos cognitivos das universitárias pesquisadas, tanto neste estudo como no de Bariani (1998). Parece ser importante que novos estudos sejam realizados com amostras diversificadas, que possam dar maior poder de generalização para a hipótese de que há diferenças relacionadas à variável gênero entre os universitários brasileiros.

No que se refere aos resultados da compreensão de leitura, constatou-se que o nível de compreensão dos universitários está aquém do esperado para estudantes nesta etapa de escolarização (Oliveira, 1993; Carpenter, Miyake \& Just, 1995; Storey, 1997). Seria esperado um nível de autonomia na compreensão de leitura em universitários, que deveriam apresentar um rendimento superior no teste de Cloze.

Não houve diferenças estatisticamente significativas entre os cursos analisados, o que é congruente com a pesquisa de Pellegrini (1996), na qual, de maneira semelhante, também não foi evidenciada tal diferença. Era esperado que os estudantes do curso de Administração apresentassem melhor nível de compreensão de leitura, em razão da quantidade de leitura de textos exigida, diferentemente dos cursos de Análise de Sistemas ou Farmácia (com maior ênfase nas aulas de laboratório). Entretanto, no estudo de Oliveira (2003) foi detectada diferença estatisticamente significativa na compreensão de leitura, sendo constatado um desempenho superior dos alunos de Psi- cologia em relação aos alunos dos cursos de Administração e Direito. Vale lembrar que na pesquisa de Oliveira todos os cursos pesquisados eram da área de humanas, o que dificulta a comparação, aqui pretendida, entre cursos de diferentes áreas do conhecimento.

Outro estudo que apontou variações na compreensão de leitura entre alunos de diferentes cursos foi o realizado por Silva e Santos, em 2004. Foi observado que alunos de Letras e Medicina tiveram pontuação mais alta do que alunos de Pedagogia, Matemática, Administração, Engenharia Civil, Psicologia e Odontologia. A autora levantou a suposição de que, por um lado, os estudantes do curso de Letras, pela própria característica do curso, teriam maior interesse pela leitura e, conseqüentemente, uma maior facilidade na compreensão. Por outro lado, analisou as informações contidas na ficha de inscrição para o vestibular, verificando que os alunos de Medicina eram oriundos de escolas particulares e de cursinhos, o que pareceu ser um elemento favorecedor de um melhor desempenho em compreensão de leitura.

A correlação entre os estilos cognitivos e a compreensão de leitura apontou um índice de correlação negativa, com nível de significância marginal, entre o estilo serialista e a compreensão de leitura. Este resultado indica uma tendência de que os estudantes que têm menor habilidade de compreensão de leitura, avaliada pelo teste de Cloze, dão maior ênfase a tópicos separados e a seqüências lógicas, buscando, posteriormente, padrões e relações no processo, para confirmar ou não suas hipóteses.

A correlação encontrada mostra que há estilos que se adaptam melhor à natureza de uma tarefa, como aponta Riding (1997) quando distingue estilo cognitivo de habilidade. Os estilos e as habilidades parecem afetar o desempenho em uma tarefa, porém, enquanto as habilidades estão relacionadas à precisão da resposta, os estilos estão relacionados à natureza da tarefa. Portanto, em relação ao estilo cognitivo, o desempenho não vai depender da capacidade e, sim, do tipo de tarefa a ser realizada.

Algumas questões podem ser exploradas no que se refere à utilização do teste de Cloze para a avaliação da compreensão de leitura. Os resultados encontrados neste estudo são compatíveis com outros descritos na literatura estrangeira (Abraham \& Chapelle, 1992; Condemarín \& Milicic, 1988; Hannon 


\section{Rosana Maria Mohallem Martins}

\& Daneman, 2001; Helfldt \& Henk, 1985) e brasileira (Bitar, 1989; Carelli \& Santos, 1998; Oliveira, 2003; Santos 1990, 1994, 1997; Silva \& Santos, 2004; Witter 1997, 1999). Assim, apontam para dificuldades expressivas apresentadas pelos universitários, sendo que a maior parte deles apresenta, como já referido, um nível de compreensão abaixo do desejável para suprir as demandas de leitura exigidas em um curso superior. Com isso, demonstram certa dependência de auxílio adicional para realizar uma leitura compreensiva. O baixo desempenho dos estudantes pôde ser detectado pelas limitações para fazer inferências e usar o conhecimento prévio, sendo essas habilidades pouco desenvolvidas em universitários ingressantes.

O investimento em pesquisas na área de avaliação psicoeducacional se faz necessário, diante das inúmeras dificuldades detectadas em relação à aprendizagem dos estudantes. Como referido, muitas variáveis internas e externas podem influenciar na aprendizagem, sendo que dentre as internas, estão os estilos cognitivos, entendidos como tendências diferenciadas no processamento da informação.

Vale ressaltar também que os dados obtidos não confirmam a idéia dos estilos cognitivos estruturados em pólos opostos, como defende Riding (1997). No entanto, aparecem de forma imbricada, como admite Messick (1984), indicando que a presença de uma dimensão de estilo não significa a ausência da outra. Este fato, no âmbito da aprendizagem, tem um valor importante, pois um aluno que possui ambas as dimensões tem maior probabilidade de ter mais êxito no desempenho escolar, adaptandose melhor às diversas condições de aprendizagem.

Há ainda que se ampliar o conhecimento sobre a identidade do construto estilo cognitivo e o de personalidade como tem sido estudado por Riding e Wigley (1997) e, no Brasil, por Santos, Sisto e Martins (2003) na pesquisa que realizaram com universitários. Tais construtos podem apresentar maior proximidade do que inicialmente se supôs.

Os resultados permitiram várias reflexões que podem ser exploradas em outras pesquisas. Quais as relações de estilos cognitivos com outras habilidades requeridas no âmbito acadêmico? Há correlação entre a compreensão de leitura e os estilos cognitivos, se eles fossem avaliados por outro tipo de instrumento (por exemplo, aqueles que os avaliam por tarefas de processamento e não por meio de auto-relato)? Há correlação entre a compreensão de leitura e estilos cognitivos em grupos específicos, como por exemplo, com estudantes hábeis ou disléxicos? Se os resultados de pesquisas demonstram que os estilos cognitivos são imbricados, por que não pesquisar essas imbricações e desenvolver um instrumento que possa avaliar os "Tipos Cognitivos"?

Em suma, este estudo procurou fornecer alguns elementos que levam ao questionamento do construto estilo cognitivo, tal como tem sido pesquisado. Além de confirmar o que tem sido investigado sobre compreensão de leitura do universitário, levantou questões, até então, pouco analisadas como a relação da compreensão da leitura com o estilo de adquirir e processar a informação. Espera-se que os resultados apresentados possam encorajar outros pesquisadores a explorar mais amplamente o tema aqui abordado.

\section{Referências Bibliográficas}

Abraham, R. G., \& Chapelle, C. A. (1992). The meaning of Cloze test scores: An item difficulty perspective. The Modern Language Journal, 76, 468-479.

Allport, G.W. (1973). Personalidade: padrões e desenvolvimento. São Paulo: Editora da Universidade de São Paulo.

Bariani, I. C. D. (1998). Estilos cognitivos de universitários e iniciação científica. Tese de Doutorado. Faculdade de Educação, UNICAMP: Campinas.

Bariani, I. C. D., \& Santos, A. A. A. (2000). Estilos cognitivos de estudantes de psicologia: Experiência em iniciação científica e séries freqüentadas. Estudos de Psicologia-Campinas, 17, 52 - 61.

Bariani, I. C. D, Sisto, F. F., \& Santos, A. A. A. (2000). Construção de um instrumento de avaliação de estilos cognitivos. Em F. F. Sisto, E. T. B. Sbardelini \& R. Primi, (Orgs.), Contextos e questões da avaliação psicológica (pp.173188). São Paulo: Casa do Psicólogo.

Bitar, M. L. (1989). Eficiência dos instrumentos de avaliação em leitura. Dissertação de Mestrado. 
Pontifícia Universidade Católica de São Paulo, São Paulo.

Bormuth, J. R. (1968). Cloze Test Readability: Criteria Reference Score. Journal Educational Measurement, 5, 189-196.

Buela-Casal, G., Carretero-Dios, H., \& Santos-Roig, M. (2001). La reflexividad-impulsividad como uma dimension continua: Validacion del sistema de classifición de Salkind y Wright (1977). Revista Latino Americana de Psicologia 33, 149-157.

Carelli, M. J. C., \& Santos, A. A. A. (1998). Condições temporais e pessoais de estudo em universitários. Psicologia Escolar e Educacional, 2, 265 - 278.

Carpenter, P. A., Miyake, A., \& Just, M. A. (1995). Language comprehension: Sentence and discourse processing. Annual Review of Psychology, 46, 91-120.

Condemarín, M., \& Milicic, N. (1988). Test de Cloze: Procedimiento para el desarrollo y la evaluación de la comprensión lectora. Santiago-Chile: Editorial Andrés Bello.

Hannon, B., \& Daneman, M. (2001). A new tool for mesuring and undertanding individual differences in the component process of reading compreehension. Journal of Educational Psychology, 93, 103-128.

Helfeldt, J. P., \& Henk, W. A. (1985). Usefulness of conventional vs total randon cued Cloze tests as measure of reading comprehension. Journal of Reading, 28, 719-725.

Henk, W. A. (1981). Effects of modified deletion strategies and scoring procedures on Cloze test performance. Journal of Reading Behavior, 13, 347-357.

Jones, A. E. (1997). Reflection-impulsivity and wholistanalytic: Two fledglings? or is R-I a cuckoo? Educational Psychology, 17 (1,2), 65-76.

Lemes, S. S. (1998). Os estilos cognitivos - dependência e independência de campo - na formação e no desempenho acadêmico em duas diferentes áreas de conhecimento: exatas e
Estilos cognitivos e compreensão leitora $\mathbf{6 7}$

humanas. Tese de Doutorado, Instituto de Psicologia da USP, São Paulo-SP.

Messick, S. (1984). The nature of cognitive style: Problems and promise in educational practice. Educational Psychologist, 19 , 59-74.

Oliveira, K. L. (2003). Compreensão de leitura, desempenho acadêmico e avaliação de aprendizagem em universitários. Dissertação de Mestrado, Programa de Estudos Pós Graduados em Psicologia, Universidade São Francisco, Itatiba SP.

Oliveira, M. H. M. A. (1993). A leitura do universitário: Estudo comparativo entre os curso de Engenharia e Fonoaudiologia da PUCAMP. Dissertação de Mestrado, Departamento de Pós Graduação em Psicologia Escolar, PUC-Campinas, Campinas-SP.

Pellegrini, M. C. K. (1996). Avaliação dos níveis de compreensão e atitudes frente a leitura em universitários. Dissertação de Mestrado, Faculdade de Ciências Humanas, Universidade São Francisco, Bragança Paulista-SP.

Perfetti, C. A. (1992). A capacidade para a leitura. Em R. Sternberg (Org.), As capacidades intelectuais humanas: Uma abordagem em processamento de informações. (pp. 72-96). Porto Alegre: Artes Médicas.

Pitta, K. B., Santos, L. A. D., Escher, C. A., \& Bariani, I. C. B. (2000). Estilos cognitivos de estudantes de psicologia: Impacto da experiência em iniciação científica. Psicologia Escolar e Educacional, 4,41-50.

Riding, R. J. (1997). On the nature of cognitive style. Educational Psychology, 17, 29-49.

Riding, R. J., \& Wigley, S. (1997). The relationship between cognitive style and personality in further education students. Personality and Individual Differences, 23, 379-389.

Rush, R., \& Klare, G. R. (1978). Re-opening the Cloze blank issue. Journal of Reading Behavior, 10, 208-210.

Santos, A. A. A. (1990). Compreensão em leitura na universidade: Um estudo comparativo entre dois 
68 Rosana Maria Mohallem Martins

procedimentos de treino. Estudos de Psicologia-Campinas, 7, 39-53.

Santos, A. A. A. (1994). Programas de remediação: Uma alternativa para o desenvolvimento da compreensão em leitores adultos. Pro-posições, 5, 114-121.

Santos, A. A. A. (1997). Psicopedagogia no terceiro grau: Avaliação de um programa de remediação em leitura e estudo. Pro-posições, 8, 27 - 37.

Santos, A. A. A., Bariani, I. C. D., \& Cerqueira, T. C. S. (2000). Estilos cognitivos e estilos de aprendizagem. Em F. F. Sisto, G. C. Oliveira, L. D. T. Fini. Leituras de psicologia para formação de professores. (pp. 44-57). Petrópolis: Vozes; Universidade São Francisco, Bragança Paulista, SP.

Santos, A. A. A., Sisto, F. F., \& Martins, R. M. M. (2003). Estilos cognitivos e personalidade: Um estudo exploratório de evidências de validade. Psico-USF, 8, 11 - 20.

Silva, M. J. M., \& Santos, A. A. A. (2004). A avaliação da compreensão em leitura e o desempenho acadêmico de universitários. Psicologia em Estudo, 9 (3), 331- 339.

Sternberg, R. J. (2000). Psicologia cognitiva. Porto Alegre: Artes Médicas.

Sternberg, R. J., \& Grigorenko, E. L. (1997). Are cognitive styles still in style? American psychologist, 52, 700-712.

Storey, P. (1997). Examining the test-taking process: A cognitive perspective on the discourse Cloze test. Language Testing, 14, 214-231.

Taylor, W.L. (1953). Cloze procedure: a new tool for measuring readability. Journalism Quarterly, 30, 415-433.

Veríssimo, L.F. (1995). Desentendimento. Ícaro Revista de bordo da Varig, XII, 136, p.13.

Vicentelli, H. (1999). Problemática de la lectura en estudiantes universitarios. Psicologia Escolar e Educacional, 3, 195-200.

Witkin, H. A., \& Goodenough, D. R. (1991). Estilos cognitivos: Natureza y orígenes. Madrid: Ediciones Pirámide.
Witter, G. P. (Org.) (1997). Leitura e universidade. Campinas: Editora Alínea.

Witter, G. P. (Org.) (1999). Leitura: Textos e pesquisas. Campinas: Editora Alínea. 